Ifo 39

RESULTS OF A NEW COMBINATION CHEYOTHERAPY (ETOPOSID-IFOSPHAMIDE-MITOXANTRONE) IN ADVANCED NHL R. Heinz,Ch.Jittrich,H.Ludwig, J.Kijhböck, G.Baumgartner,J.Schüler, R. Waldner, M. Wirth (Vienna Lymphoma Study aroup)

During the last 18 months we treated 45 patients with a new combination: Ifosphamid $1 \mathrm{~g}, \mathrm{VP}-16$ loomg, Witoxantrone $4 \mathrm{mg} / \mathrm{m}^{2}$, Prednisone $100 \mathrm{mg}$. These cytostatic agents were given day 1-3, Prednisone was tapered gradually. Bleomycin 15 U was given i.r. on day 15.Cycles were repeated every 3 weeks. 30 patients ( $L B$. 3, IB 9, CB 6,CC 4,Cb-CC 7, Lennert's Lyimphoma 1) received this combination as salvage therapy. Two third of these patients had never achieved a stable CR with various forms of pretreatirent including anthracycline containing regimens. All had advanced stage of disease.objective response was seen in 15 patjents $(5 \mathrm{CR}$, Io $P R$. In addition 7 patients had minor responses lasting up to 8 inunths. Because of these results in pretreated patients we introduced VIil-schedule in 15 untreated patients (LB 1, IB $3, C B 5, C B-C C 3$, IC 1,pleomorphic T-cell lymphama 2). Al I but 2 patients had advanced disease.objective response was seen in 8 of 10 evaluable patients. 2 patients (pleomorphic T-cell lymphoma) did not respond, in 3 patients is too early to evaluate. Duration of response was rather short. Subjective tolerance was excellent.Dose limiting toxicity was granulocyte depression.

Ludwig Boltzmann-Institut für Leukämieforschung und Hämatologie und 3. Medizinische Abtejlung, Hanusch Krankenhaus, Heinrich Coll in Strasse 30 , A-1140 wien

\section{Epi 01}

EPIRUBICIN IN METASTASIZED BREAST-CANCER

J. H. HartTapp

Patients with systemic metastasized breast-cancer are uncurable. Remissions with longer survival can be induced by chemotherapy in 50 to $80 \%$ of the cases with 10 to $20 \%$ complete remission, although the recurrence is unavaidable. Therefore the strateay of therapy in breast-cancer must include two aspects. First prolongation of overall survival by multiple remissions with renimens that are not crossresistant and secondly conserve the quality of life by minimization of therapy conditioned side-effects.

EPIRUBICIN, the new anthracyclin derivative and analonue of DOXORUBICIN (probably the most active chemotherapeutic agent aqainst breast cancer) exhibits the same hich activity but lower side-effects compared with the parent compound.

Complete and partial remissions in $33 \%$ of 313 breastcancer patients could be achieved with EPIRliBICIN. In three other studies the efficacy and side-effects of EPIRUBICIN were compared with the established drug DOXORUBICIN. The remission rate was nearly the same but side-effects such as nausea, vomiting, stomatitis, bone marrow toxicity and congestive heart failure were lower. In a randomized multicentre study, 520 patients were treated with EPIRUBICIN or DOXORUBICIN in combination with CYCLOPHOSPHAMIDE and FLUOROURACIL. The remission rates were 52 vs. $54 \%$ respectively, but the toxicity of the EPIRUBICIN combination group was significantiy lower.

Medizinische Universitätsklinik, Onkologie, SiegmundFreud-Str. 25, 5300 Bonn-Venusberg
Epi 02

THE_ERENCH_FAC_YS_FEC_STUDY_IN_ADYANCED_BREAST_GANCER

R, KEILING, P. P. ARHAND, P. HURTELOUP, P. CA PPELAERE In behalf of the FRENCH EPIRUBICIN STUDY GROUP On the basis of the results obtalned in the Eptrubicin Phase II trial conducted by the EORTC Cliaical Screening Group (1 CR, 8 PR aut of 28 patients and 6 PR in 11 untreated patients with no major cardiotoxicity) the French Epirubicin Study Group has decided to run a Phase IrI trial comparing Epirubicin (Ept) to Doxorubieln (DX) IE a FAC conbination For advanced breast cancer In Harch 1982. According the preclinical and phase II studies the 2 athracyclines were compared on an equieclar dosage of $50 \mathrm{mg} / \mathrm{m}^{2}$ combined with 5 FU and Cytoxan both at $500 \mathrm{mg} / \mathrm{m}^{2}$. The three drugs were administered on day 1 and cycles were repeated at a 3 weeks interval. The maximal cumulative - dose was $550 \mathrm{mg} / \mathrm{m}^{2}$ for $\mathrm{DX}$ and $700 \mathrm{mg} / \mathrm{m}^{2}$ for $\mathrm{Epi}$.

Ains of this prospective randouized st udy was : to coupare the efficacy of the two combinations chemotherapy, FEC vs FAC and also the qualitative and the quantitative aspects (in particular cardiotoxicity) associated th these treatments. Therapeutics re. sponses evaluated according the wHO criterla are isted in table 1 .

\begin{tabular}{|lll|} 
& FAC & FEC \\
\hline Complete Response (CR) & 12 & 13 \\
Partlel Response (PR) & 49 & 44 \\
No Change & 38 & 41 \\
Progression & 19 & 22 \\
Response rate (CR + PR) & $52( \pm 9) \%$ & $48( \pm 9) \%$ \\
Table I : Response rate by treatment arm. &
\end{tabular}

Conclusions of this phase 3 trial are:

- There is no difference concerning response rate, time to maximal response, duration of response between EPI and DX In this combination - FEC is significantly less toxic than FAC regarding nyelotoxicity. a lopecia and gastro-intestinal toxictty.

- There are 3 congestive heart lailures in the Doxorublcin regimen, none in the Eplrubicin regimen despite the higher cumulative dose. On the basis of the alove results it appears reasonable: - to replace Doxorubiein by Eplrubicin at equimolar dosis in the chemotherapy regimen under study.

\section{Epi 03}

EPICO in the Treatment of Small Cell Lung Cancer

- Interim Analysis -

P. Orings, H. Bülzebruck, D. Hruska*, H.G. Manke und G. Schuler Krankenhaus Rohrbach, Klinik für Thoraxerkrankungen der LVA Baden, Heidelberg "f achklinik Schillerhöhe Cerling̣en

Patients: 59 patients with untreated histalogically proven small cell lung cancer were entered into a phase II study. All patieints haf measurable tumor. The patients underwent a classical staging procedure before entering the study, including chest $X$-ray, bronchoscopy, tomograms of livet and brain, bone scan, bone marrow biopsy, and blood chemistry. The courses of

Iable 1: Characteristics of the patients: performance status

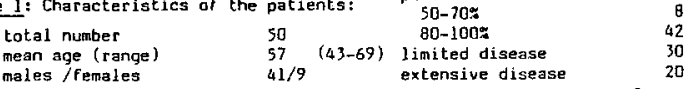
Ireatment: The therapeut ic regimen consited of epidoxirubicine $70 \mathrm{mg} / \mathrm{m}^{2}$ on day 1 , cyclophosphamide $1000 \mathrm{mg} / \mathrm{m}^{2}$ on day 1 , and vincristine $2 \mathrm{mg}$ on day 1 . recovery. Evaluat icn of response was wees, according to hematologic to criteria established from EOR IC and $\mathrm{NCI}$.

Results: As a whole, the response rate of these preliminary results was $62 \%$, with $16 \% \mathrm{CR}(9 / 50)$ and $46 \mathrm{PR}(23 / 50)$. Table 2 shows the distribution of response according to possible prognost ic factors.

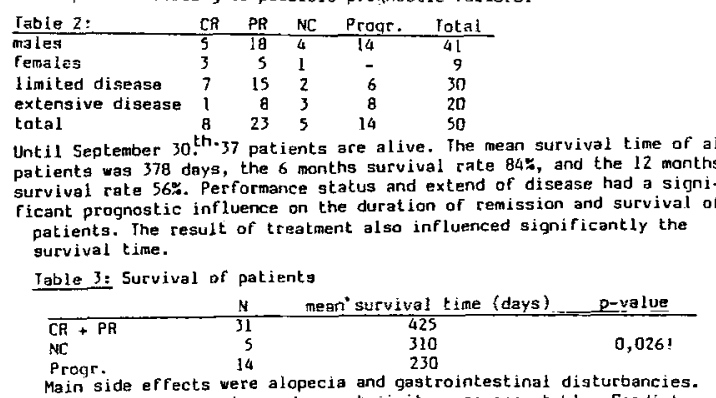

Main side effects were alopecia and gastrointestinal disturbancies. done martru depressian and

Conclusion: These preliminary results demonstrate the activity of EPICO 